\title{
Questionário de Perceção de Competências Académicas: Resultados do Estudo Exploratório
}

\author{
Sofia de Lurdes Rosas da Silva*, Joaquim Armando Gomes Ferreira**, António Gomes Ferreira** \\ *Escola Superior de Educação - Instituto Politécnico de Coimbra, CEIS XX, Portugal **Faculdade de Psicologia e de Ciências da \\ Educação da Universidade de Coimbra, CEIS XX, Portugal
}

\begin{abstract}
Resumo
Quando se analisa a literatura sobre ensino superior, o domínio da aquisição de conhecimentos e competências disciplinares e académicas fundamentais tem merecido destaque. O objetivo desta investigação prendeu-se com o desenvolvimento de um questionário de autoavaliação de competências académicas (Questionário de Perceção de Competências Académicas - QPCA) alcançadas durante os anos de ensino superior, considerando as características culturais/educativas do Politécnico. Assim, partimos da análise de conteúdo de entrevistas realizadas junto de 31 estudantes e do seu confronto com a literatura. A versão final do QPCA resultou numa solução de um fator, com um nível de consistência interna bastante aceitável $(\alpha=.91)$.

Palavras chave: QPCA, construção, estudo exploratório
\end{abstract}

\section{Introdução}

Quando se analisa a vasta literatura sobre o ensino superior, seja numa perspetiva mais desenvolvimentista ou mais contextualista, a par do desenvolvimento de competências intelectuais, há um outro domínio que é impreterivelmente considerado como fundamental e, em virtude desse estatuto, se constitui num dos grandes objetivos ou missões da educação superior: aquisição e aprofundamento de conhecimentos e competências em determinadas áreas disciplinares e/ou vocacionais e competências académicas gerais ou fundamentais, tais como capacidades de compreensão na leitura, de escrita, e de cálculo (Pascarella \& Terenzini, 1991, 2005; Silva, 2012). Não significa que outros domínios do desenvolvimento sejam desconsiderados quanto à importância que representam para o indivíduo e as sociedades, mas também é um facto que estes dois domínios se constituíram e ainda se constituem no alvo principal do interesse de políticos, administradores de instituições educativas, professores, técnicos e serviços de apoio ao estudante, e de entidades empregadoras (Silva, 2012).

$\mathrm{O}$ interesse pela maximização da aprendizagem, das competências académicas e das competências nos modos de pensar, aliado à crença, recorrente na literatura e nos discursos políticos, de que os contextos de ensino superior ricos se constituem em oportunidades únicas para potenciar a mudança, tem conduzido ao desenvolvimento de modelos teóricos e de estudos, cujos objetivos se centram, entre outros: (1) em analisar até que ponto os estudantes atingem os objetivos propostos; e (2) em delinear intervenções contextualizadas que propiciem as condições necessárias e mais adequadas à sua concretização ou desenvolvimento (e.g Kuh, Vesper,
Connolly, \& Pace, 1997; National Study of Student Engagement, 2000, 2001, 2002, 2003, 2004, 2005, 2007; Sax, Astin, Korn, \& Mahoney, 1997).

Em consequência, muitos têm sido os instrumentos desenvolvidos que procuram averiguar se os programas e práticas institucionais estão ou não a produzir os efeitos desejados (e.g. College Student Experiences Questionnaire, The College Student Report).

Apesar de ser reconhecido que as práticas institucionais podem potenciar os níveis de desenvolvimento do estudante, vem sendo defendido que essas práticas devem ser analisadas à luz das especificidades dos contextos, em particular do modo como estas articulam as práticas educativas com a missão e filosofia institucionais (Kuh et al., 1991, 2005). Tal implica necessariamente instrumentos de avaliação que respeitem a cultura da instituição (Silva, 2012).

Face a este enquadramento, o objetivo principal desta investigação foi o de construir o Questionário de Perceção de Competências Académicas (QPCA), considerando as características culturais e educativas de uma instituição do ensino superior politécnico português, e efetuar o estudo das suas qualidades psicométricas (dimensionalidade e consistência interna).

\section{Método}

Como referido anteriormente, o objetivo desta investigação consistiu na construção de um instrumento de autoavaliação de competências académicas (QPCA) adaptado às características culturais da instituição escolhida para fazer o estudo. Para concretizar este objetivo efetuou-se uma entrevista a 31 estudantes de uma instituição do Ensino Superior Politécnico sobre que competências académicas consideravam que deveriam adquirir durante o seu percurso no ensino superior e aquelas que efetivamente consideravam estar a adquirir.

Para a construção do instrumento partiu-se dos resultados da análise de conteúdo e do confronto das categorias que foram emergindo dessa análise com os principais resultados da investigação no domínio das competências académicas.

A primeira versão do intrumento foi testada com um grupo de 45 estudantes e o estudo exploratório, após alguns ajustes ao QPCA, foi conduzido com uma amostra de 576 estudantes.

Os pontos que se seguem referem-se aos procedimentos adotados e apresentam os estudos de validação utilizados. 


\section{Sujeitos}

No pré-teste do QPCA participaram 45 estudantes (7 do sexo masculino e 38 do feminino), a frequentar o $1 .^{\circ}$ $(n=24), 2 .^{\circ}(n=9)$ e $3 .^{\circ}$ ano $(n=12)$, de diversos cursos de formação inicial (Arte e Design, Animação Socioeducativa, Comunicação e Design Multimédia, Comunicação Organizacional, Comunicação Social, Desporto e Lazer, Gerontologia Social, Língua Gestual Portuguesa, Música), com idades compreendidas entre os 17 e os 44 anos.

No estudo exploratório participaram 576 estudantes, do $1 .^{\circ}(\mathrm{n}=195), 2 .^{\circ}(\mathrm{n}=187)$ e $3 .^{\circ}$ ano $(\mathrm{n}=194)$. De salientar que a amostra, maioritariamente do sexo feminino $(\mathrm{n}=403 ; 70 \%)$, reflete as características do contexto de estudo. Os estudantes do sexo masculino são mais velhos em média um ano $(\mathrm{M}=21.57 ; \mathrm{DP}=3.75)$ do que os do sexo feminino $(\mathrm{M}=20.59 ; \mathrm{DP}=2.80)$, diferença de idades considerada significativa $(\mathrm{t}=2.497 ; \mathrm{p}<.05)$.

\section{Procedimento}

Como referido, a construção do QPCA partiu da análise de conteúdo das entrevistas realizadas aos estudantes e do confronto das categorias que foram emergindo dessa análise com os principais resultados da investigação no domínio. Da análise de conteúdo emergiram duas categorias: competências fundamentais e competências vocacionais. O confronto das fontes levou-nos a partir do pressuposto da possibilidade de bidimensionalidade do construto.

Para avaliar o construto foram elaborados itens que, na sua maioria, emergiram do conteúdo das entrevistas realizadas. Procurou-se, nesta fase, elaborar-se o maior número de itens possível, para selecionar posteriormente os melhores itens a reter (Freire \& Almeida, 2001). Procedeu-se ainda a uma comparação com a literatura com o objetivo de refinar e completar o instrumento. Assim, a versão inicial do instrumento continha alguns itens deduzidos a partir de estudos e teorias apresentadas por outros investigadores.

Neste processo também tiveram de ser tomadas decisões relativamente ao formato de resposta. A opção recaiu sobre a escala de likert de cinco pontos (não se aplica, aplica-se pouco, aplica-se moderadamente, aplica-se muito, aplica-se totalmente).

Depois de elaborado, o QPCA foi inicialmente submetido a um pré-teste junto de um grupo de 45 estudantes, com os seguintes objetivos: 1) avaliar o nível de compreensibilidade das questões; 2) detetar questões inúteis ou redundantes; 3) identificar perguntas relevantes que estivessem ausentes; e 4) avaliar a qualidade gráfica do instrumento. Depois de se solicitar o preenchimento dos instrumentos e de se pedir que os estudantes assinalassem no próprio instrumento dificuldades ou sugestões à medida que os fossem preenchendo, procedeu-se a uma reflexão falada dos itens. Na generalidade, os estudantes consideraram não ter dúvidas e terem compreendido bem o que se lhes era pedido.

Elaborada a versão final do QPCA, procedeu-se à sua administração junto de uma amostra de 576 estudantes com o objetivo de realizar um estudo exploratório que permitisse não apenas reduzir a extensão do instrumento, como também avaliar a sua qualidade psicométrica, em particular a sua dimensionalidade e respetiva consistência interna. Para o efeito, efetuaram-se análises fatoriais exploratórias (método de extração em componentes principais, rotação ortogonal varimax) e análises da consistência interna através do alpha de Cronbach. No ponto que se segue apresentam-se os resultados.

\section{Resultados}

A versão inicial do QPCA era constituída por 15 itens distribuídos por 2 dimensões (cf. Quadro 1).

Quadro 1.

Dimensões e itens da versão inicial do QPCA

\begin{tabular}{|c|c|}
\hline Dimensão & Item \\
\hline \multirow{6}{*}{$\begin{array}{l}\text { Competências } \\
\text { Académicas } \\
\text { Fundamentais }\end{array}$} & $\begin{array}{l}\text { 1. Sinto que desenvolvi as minhas } \\
\text { competências de cálculo }(+)\end{array}$ \\
\hline & $\begin{array}{l}\text { 3. Sei fazer uma pesquisa na biblioteca ou } \\
\text { na internet }(+)\end{array}$ \\
\hline & 5. Noto que escrevo melhor $(+)$ \\
\hline & 7. Sinto que falo melhor a minha língua $(+)$ \\
\hline & $\begin{array}{l}\text { 9. Aprendi a distinguir um bom trabalho de } \\
\text { um mau }(+)\end{array}$ \\
\hline & $\begin{array}{l}\text { 11. Sinto que tenho crescido ao nível da } \\
\text { qualidade dos meus trabalhos }(+)\end{array}$ \\
\hline \multirow{9}{*}{$\begin{array}{l}\text { Competências } \\
\text { Vocacionais }\end{array}$} & $\begin{array}{l}\text { 2. Sinto-me capaz de fazer coisas que } \\
\text { aprendi no curso }(+)\end{array}$ \\
\hline & $\begin{array}{l}\text { 4. Não me sinto preparado para entrar no } \\
\text { mercado de trabalho (-) }\end{array}$ \\
\hline & $\begin{array}{l}\text { 6. Acredito que as aprendizagens que fiz, } \\
\text { me permitirão ser um profissional } \\
\text { competente }(+)\end{array}$ \\
\hline & $\begin{array}{l}\text { 8. Alarguei os meus horizontes em relação à } \\
\text { minha área de formação }(+)\end{array}$ \\
\hline & $\begin{array}{l}\text { 10. Consegui esclarecer o que é a minha } \\
\text { área de formação e explorar alternativas }(+)\end{array}$ \\
\hline & $\begin{array}{l}\text { 12. Aprendi a ver a ligação entre a teoria e a } \\
\text { prática profissional }(+)\end{array}$ \\
\hline & $\begin{array}{l}\text { 13. Aprendi técnicas e ferramentas } \\
\text { importantes para a minha profissão }(+)\end{array}$ \\
\hline & $\begin{array}{l}\text { 14. Adquiri e aprofundei conhecimentos } \\
\text { profissionais }(+)\end{array}$ \\
\hline & $\begin{array}{l}\text { 15. Sinto-me preparado para encarar } \\
\text { alternativas profissionais que ná } \\
\text { exclusivamente da área do curso }(+)\end{array}$ \\
\hline
\end{tabular}

Estes 15 itens pretendiam avaliar a perceção de aquisição de um conjunto de competências em dois domínios: competências fundamentais, constituída por 6 itens (cálculo, língua materna oral e escrita, pesquisa e qualidade do trabalho académico), e competências vocacionais, constituída por 9 itens (competências vocacionais, preparação para o mercado de trabalho, conhecimento mais alargado sobre a área de formação, aquisição de conhecimentos e ferramentas vocacionais). Resultados elevados nestas subescalas traduzem um sentimento de aquisição de conhecimentos e competências académicas transversais e competências académicas vocacionais.

Numa primeira fase do estudo, para os 15 itens que avaliavam a Perceção de Competências Académicas, os indicadores fornecidos pelo teste KMO 
[Kaiser-Meyer-Olkin Measure of Sampling Adequacy $=.929]$, pelo teste de esfericidade de Bartlet [ $\chi^{2}(105)=$ 4346, 319, p <.000] e pela matriz anti-imagem (com valores na diagonal principal a oscilar entre .80 e .96) revelaram-se adequados à prossecução da análise fatorial.

Os estudos preliminares com uma análise fatorial em componentes principais, rotação ortogonal Varimax, revelaram 3 fatores que no total explicavam $61.4 \%$ da variância. $\mathrm{O}$ primeiro fator agrupava 13 dos 15 itens. $\mathrm{O}$ segundo fator agrupava o item 1 e o terceiro fator o item 4. A observação do gráfico de progressão dos valores próprios (scree test) sugeriu a organização em 1 fator, solução que se mostrou interpretável, apesar do pressuposto inicial de bidimensionalidade.

No processo de organização e determinação do fator foram considerados os seguintes critérios (Hair, Black, Babin, Anderson, \& Tatham, 2006):

1. Critério de Kaiser (com valor próprio superior a 1);

2. Peso fatorial dos itens igual ou superior a .30 ;

3. Consistência interna, medida pelo alpha de Cronbach, superior a .70

4. Percentagem de variância explicada do fator não inferior a $40 \%$.

5. Valor de comunalidades superior a .30;

6. Ponderação dos critérios estatísticos em função do valor conceptual do item para o construto.

No processo de organização dos dados foram eliminados itens com base nos seguintes critérios, ponderados em função do contributo conceptual do item: valor das comunalidades abaixo de .30 (eliminado os itens 4 e 1); valor de saturação no fator abaixo de .30 (eliminado o item 1). Mantiveram-se os itens 3 e 5 com valores de comunalidades inferiores a .30 , dado o seu valor conceptual para a definição do construto.

Foi realizada a Análise Fatorial em Componentes Principais, rotação ortogonal Varimax, com o conjunto dos 13 itens. Os indicadores fornecidos pelo teste KMO $[K M O=.931]$ e pelo teste de esfericidade de Bartlet $[\chi 2$ $(78)=4195,116, p<.000]$ indicaram a viabilidade da análise fatorial, o mesmo acontecendo com a matriz anti imagem com valores na diagonal principal que oscilaram entre .82 e .97 .

A solução encontrada evidenciou uma estrutura fatorial organizada em um fator com um valor próprio de 6.67 que explica $51.3 \%$ da variância. Os valores das comunalidades $\left(\mathrm{h}^{2}\right)$ registadas nos 13 itens variaram entre $.25<\mathrm{h}^{2}<.67$. Observando o Quadro 2 verifica-se que o fator 1 apresenta saturações que variam entre $.50 \mathrm{e}$ .81 .

Quadro 2.

Análise Fatorial dos 13 itens do QPCA

\begin{tabular}{|c|c|c|}
\hline Itens & Fator & $\mathrm{h}^{2}$ \\
\hline QPCA - 14 & .81 & .67 \\
\hline QPCA - 12 & .81 & .66 \\
\hline QPCA - 13 & .81 & .66 \\
\hline QPCA - 11 & .79 & .63 \\
\hline
\end{tabular}

\begin{tabular}{|l|c|r|}
\hline QPCA - 10 & .78 & .62 \\
\hline QPCA - 8 & .78 & .62 \\
\hline QPCA - 6 & .76 & .59 \\
\hline QPCA - - & .68 & .47 \\
\hline QPCA - 9 & .68 & .46 \\
\hline QPCA - 15 & .62 & .39 \\
\hline QPCA - 7 & .58 & .34 \\
\hline QPCA - 5 & .52 & .27 \\
\hline QPCA - 3 & .50 & .25 \\
\hline \multicolumn{2}{|c|}{ Valor próprio $=6.67 ;$ Variância explicada $=51.3 \%$} \\
\hline
\end{tabular}

Quanto ao índice de consistência interna, uma análise dos itens permite verificar que a generalidade se correlaciona com a escala total, em valores superiores a .30, valor mínimo a reter segundo Cronbach (1984) (cf. Quadro 3). O valor total obtido revelou-se muito bom $(\alpha=.91)$.

Quadro 3.

Consistência interna (alpha de Cronbach) do QPCA

\begin{tabular}{|c|r|r|r|r|}
\hline Item & Média & $\begin{array}{c}\text { Desvio- } \\
\text { Padrão }\end{array}$ & $\begin{array}{c}\text { Correlação } \\
\text { Item-total }\end{array}$ & $\begin{array}{c}\alpha \text { com } \\
\text { exclusão } \\
\text { do item }\end{array}$ \\
\hline 2 & 3.77 & .75 & .61 & .90 \\
\hline 3 & 4.16 & .72 & .43 & .91 \\
\hline 5 & 3.38 & .93 & .47 & .91 \\
\hline 6 & 3.72 & .82 & .71 & .90 \\
\hline 7 & 3.46 & .92 & .54 & .91 \\
\hline 8 & 3.95 & .78 & .72 & .90 \\
\hline 9 & 3.85 & .79 & .62 & .90 \\
\hline 10 & 3.84 & .76 & .72 & .90 \\
\hline 11 & 3.87 & .75 & .73 & .90 \\
\hline 12 & 3.83 & .74 & .76 & .90 \\
\hline 13 & 3.84 & .73 & .75 & .90 \\
\hline 14 & 3.83 & .77 & .75 & .90 \\
\hline 15 & 3.63 & .86 & .55 & .91 \\
\hline & \multicolumn{5}{|c}{} \\
\hline
\end{tabular}

\section{Discussão}

Neste artigo apresentam-se os procedimentos adotados para a construção do QPCA e os dados psicométricos do estudo exploratório. Efetuou-se uma Análise Fatorial Exploratória para averiguar as componentes principais do QPCA. Apesar do pressuposto inicial de bidimensionalidade, a solução fatorial encontrada de um fator que explica 51,3\% da variância, pareceu-nos com sentido conceptual.

Considerando a literatura (Hair et al., 2006), o facto de termos encontrado correlações item-total do fator superiores a .50 para a maioria dos itens é um indicador de existência de níveis de consistência interna bastante aceitáveis. A exceção verificou-se apenas para os itens 3 e 5 .

No que concerne aos resultados da comunalidades quase todos os itens atingem valores acima de .30 . A exceção verifica-se para os itens 5 e 3, mas dado o seu valor cenceptual, optou-se por mantê-los.

Quanto aos pesos dos itens que constituem o fator, verificamos que todos os itens apresentam pesos fatoriais aceitáveis (iguais ou superiores a .50) (Hair et al., 2006). 
O valor do alpha de Cronbach encontrado para o fator $(\alpha=.91)$ traduz uma muito boa consistência interna (Hill \& Hill, 2009).

Os resultados obtidos neste estudo exploratório são reveladores das boas qualidades psicométricas deste instrumento.

É nosso entendimento que a construção e validação de instrumentos de medida fundados na cultura das instituições de ensino superior, que permitam avaliar os efeitos das experiências educativas e da sua articulação com a missão e filosofia institucionais, se assume de especial relevância. De facto, para se implementarem medidas com vista à promoção de experiências educativas de qualidade e significativas do ponto de vista do desenvolvimento do estudante, torna-e condição fundamental o conhecimento e compreensão da cultura institucional, das suas potencialidades e fragilidades.

Neste contexto, esperamos que o Questionário de Perceção de Competências Académicas se possa constituir num desses instrumentos de monitorização no domínio da aquisição e desenvolvimento de competências académicas por parte dos estudantes.

Para finalizar, recomendamos que se efetuem estudos que confirmem a dimensionalidade do QPCA.

\section{Referências}

Cronbach, L. (1984). Essentials of psychological testing ( $4^{\text {th }}$ ed.). New York: Harper \& Row.

Freire, T., \& Almeida, L. (2001). Escalas de avaliação, construção e validação. In E. Fernandes \& L. Almeida (Eds.), Métodos e técnicas de avaliação: Contributos para a prática e investigação psicológicas (pp. 109-128). Braga: Universidade do Minho, Centro de Estudos em Educação e Psicologia.

Hair, J., Anderson, R., Tatham, R., \& Black, W. (2006). Multivariate data analysis ( $6^{\text {th }}$ ed.). New Jersey: Pearson Education, Inc.

Hill, M., \& Hill, A. (2009). Investigação por questionário. Lisboa: Edições Sílabo.

Kuh, G., Vesper, N., Connoly, M., \& Pace, R. (1997). College students experience questionnaire. Bloomington: Indiana University Center for Postsecondary Research and Planning.

Kuh, G., Schuh, J., Whitt, E., Andreas, R., Lyons, J., Strange, C., Krehbiel, L., \& MacKay, K. (1991). Involving colleges: Successful approaches to fostering student learning and development outside the classroom. San Francisco: Jossey-Bass.

Kuh, G., Kinzie, J., Schuh, J., Whitt, E., \& Associates (2005). Student success in college: Creating conditions that matter. San Francisco: Jossey-Bass.

National Survey of Student Engagement (NSSE). (2000). The NSSE 2000 report: National Benchmarks of effective educational practice. Bloomington, IN: Indiana University Center for Postsecondary Research.

National Survey of Student Engagement (NSSE). (2001). Improving the college experience: National Benchmarks of effective educational practice. Bloomington, IN: Indiana University Center for Postsecondary Research.
National Survey of Student Engagement (NSSE). (2002). From promise to progress: How colleges and universities are using student engagement results to improve collegiate quality. Bloomington, IN: Indiana University Center for Postsecondary Research.

National Survey of Student Engagement (NSSE). (2003). Converting data into action: Expanding the boundaries of institutional improvement. Bloomington, IN: Indiana University Center for Postsecondary Research.

National Survey of Student Engagement (NSSE). (2004). Student engagement: Pathways to collegiate sucess. Bloomington, IN: Indiana University Center for Postsecondary Research.

National Survey of Student Engagement (NSSE). (2005). Student engagement: Exploring different dimensions of student engagement. Bloomington, IN: Indiana University Center for Postsecondary Research.

National Survey of Student Engagement (NSSE). (2007). Experiences that matter: Enhancing student learning and sucess. Bloomington, IN: Indiana University Center for Postsecondary Research.

Pascarella, E., \& Terenzini, P. (1991). How college affects students: findings and insights from twenty years of research. San Francisco: Jossey-Bass.

Pascarella, E., \& Terenzini, P. (2005). How college affects students: a third decade of research. San Francisco: Jossey-Bass.

Sax, L., Astin, A., Korn, W., \& Mahoney, K. (1996). The American Freshman: National Norms for Fall 1995. Los Angeles: Higher Education Research Institute, UCLA.

Silva, S. (2012). Dinâmicas de envolvimento e de desenvolvimento do estudante do ensino superior. Tese de Doutoramento apresentada à Faculdade de Psicologia e de Ciências da Educação da Universidade de Coimbra, Coimbra, Portugal. 